\title{
PROMOÇÃO E EDUCAÇÃO EM SAÚDE COM ADOLESCENTES: RELATO DE CASO
}

Paloma Cinthia Duarte Silva ${ }^{1}$, Luiz Henrique Batista Monteiro², Tainara Catozzi Denardi $^{3}$, Ivânia Vera ${ }^{4}$, Roselma Lucchese ${ }^{5}$

${ }^{1}$ Acadêmica do curso de enfermagem da Universidade Federal de Goiás, Regional

Catalão, Catalão, Goiás, Brasil. palomacinthia1@hotmail.com

${ }^{2}$ Acadêmico do curso de enfermagem da Universidade Federal de Goiás, Regional

Catalão, Catalão, Goiás, Brasil.

${ }^{3}$ Acadêmica do curso de enfermagem da Universidade Federal de Goiás, Regional

Catalão, Catalão, Goiás, Brasil.

${ }^{4}$ Docente do curso de enfermagem da Universidade Federal de Goiás, Regional

Catalão, Catalão, Goiás, Brasil.

${ }^{5}$ Docente do curso de enfermagem da Universidade Federal de Goiás, Regional

Catalão, Catalão, Goiás, Brasil.

Recebido em: 03/10/2016 - Aprovado em: 21/11/2016 - Publicado em: 05/12/2016

DOI: 10.18677/EnciBio_2016B 190

Este é um estudo descritivo tipo relato de experiência que objetivou descrever os resultados alcançados a partir da experiência de promoção e educação em saúde com adolescentes de uma instituição pública de ensino na região Centro-Oeste do Brasil. Considerando os complexos processos de transformação e de decisão, a influência familiar e de amigos, e, a vulnerabilidade presentes no período da adolescência, realizou-se oficinas com adolescentes, desenvolvidas por acadêmicos de enfermagem, estagiários em uma Unidade Básica de Saúde/Estratégia Saúde da Família, no período de maio a junho de 2016. Para a execução das oficinas empregou-se a metodologia participativa, tendo em vista que esta abordagem permite 0 envolvimento do sujeito no processo da construção do conhecimento. Os resultados demonstraram que os jovens envolvidos nas oficinas tinham insuficiência de informações de saúde, assim, as ações desenvolvidas se mostraram como uma ferramenta conveniente e eficaz para promover mudanças benéficas, diminuir suas vulnerabilidades e auxiliar no bom enfrentamento das dificuldades presentes nesse período da vida. Percebeu-se ainda, que a escola é um ambiente favorável para a execução de atividades desse cunho, na medida em que, por ser um local onde os adolescentes passam grande tempo do dia, o alcance dos objetivos se torna mais fácil.

PALAVRAS-CHAVE: Adolescente; Educação em saúde; Enfermagem.

\section{PROMOTING AND EDUCATION IN HEALTH WITH TEENAGERS: EXPERIENCE REPORT}

\footnotetext{
ABSTRACT

This is a descriptive study of a experience report that aimed to describe the achieved objectives as of experience from promoting and education in health with ENCICLOPÉDIA BIOSFERA, Centro Científico Conhecer - Goiânia, v.13 n.24; p.1853 
teenagers of an educational public institution in the central-west region of Brazil. Considering the complex processes of transformation and the decision, the family influence as well as friend's and the vulnerability exposed in the stage of adolescence, workshops were carried out with the teenagers, developed by nursing academics in their intern period on a health unit/Family Health Strategy, from May to June of 2016. To carry out the workshops it was used the participatory methodology, keeping in mind that this approach allows the subject engagement in the process of knowledge construction. The results demonstrated that the youth group involved in the workshops had insufficient information about health, so, the actions developed showed as convenient tool and effective to promote benefic changes, reducing its vulnerability and assisting in confronting the present difficulties in this stage of life. It was possible to see that the school is a friendly environment to execute the activities of such mark, as it is a place where the teenager spend great part of the day, the objectives become easily achievable.

KEYWORDS: Teenagers; Educational health; Nursing

\section{INTRODUÇÃO}

A adolescência é a fase da vida compreendida entre os 10 e 19 anos de idade, segundo a Organização Mundial da Saúde (OMS, 2016). Essa faixa etária constitui um momento de descobertas e de diversas transformações do sujeito, tanto do ponto de vista comportamental, quanto na esfera psicológica e social. Porém, tal conceito não se faz de maneira efetiva na realidade vivida por muitos adolescentes, que estão constantemente vulneráveis a diversas situações (CEDARO et al., 2012).

Vulnerabilidade envolve fragilidade e dependência, e afeta diretamente a saúde dos adolescentes, especialmente aqueles de menor nível socioeconômico. A fragilidade e a dependência de adultos submete esse público aos problemas presentes no ambiente físico e social nos quais se encontra, dessa forma, mesmo não havendo doença, as perturbações psicológicas, mentais e sociais por si só, são capazes de alterar o estado de saúde (SIERRA \& MESQUITA, 2006). Partindo disso, é crucial assumir o desafio de promover educação, como forma de atender as demandas contemporâneas e sensibilizar sobre as alternativas de prevenção de agravos, possibilitando o empoderamento do sujeito para o planejamento e execução das ações (COSTA et al., 2015).

O estabelecimento de ações de articulação transversal que enfatizam os fatores de risco aos quais a população é exposta, considerando as singularidades e reais necessidades, territórios e culturas existentes no Brasil, define o termo Promoção da Saúde (PS). É uma estratégia que objetiva reduzir a vulnerabilidade, incentivar a equidade e suscitar a participação e o controle social na gestão das políticas públicas (BRASIL, 2007).

A realização de atividades e projetos que geram benefícios na vida e no bem- estar dos indivíduos envolve a participação de vários seguimentos da sociedade civil, visto que a PS é um ato de corresponsabilidade social. No setor da educação, as escolas desempenham um papel fundamental e, quando articuladas aos profissionais da saúde, organizações não governamentais, sociedade de bairro e com os próprios alunos, fomentam a prática de hábitos saudáveis e a prevenção de atitudes nocivas à saúde (COSTA et al., 2013).

Desse modo, promover educação em saúde, estendida como uma ação de PS é uma forma de conscientizar e prevenir os principais agravos que acometem 
os educandos no período escolar, entre eles, o consumo de álcool, tabaco e outras drogas, a morbimortalidade por violência e acidentes, além da gravidez precoce e as Doenças Sexualmente Transmissíveis (DST). Para que essas ações sejam efetivas, é necessário que haja coesão entre o que é ensinado por profissionais da educação e da saúde, no intuito de proporcionar melhor entendimento por parte dos adolescentes (BRASIL, 2009).

Por educação em saúde, entende-se um método que constrói saberes, visa o domínio de temas relacionados à saúde, a autonomia e o alcance dos direitos de cidadania da população (FALKENBERG et al., 2014). Envolve a abordagem de aspectos capazes de favorecer um futuro melhor aos jovens, e, apesar de não garantir que as mudanças sejam imediatas, oportuniza reflexão sobre as ações futuras. Assim, a inserção da educação em saúde no contexto escolar se torna uma demanda social, na qual os debates e a construção compartilhada do conhecimento respondem como estratégias prioritárias (COSSA \& JARDIM, 2011; COUTINHO et al., 2015).

Ao considerar os complexos processos de transformação e de decisão, a influência familiar e de amigos, e, a vulnerabilidade presentes no período da adolescência é indispensável valorizar as intervenções em saúde como a do presente estudo, uma vez que as mudanças que ocorrem em tal período da vida podem gerar impactos no âmbito da saúde pública (BRASIL, 2009). Além disso, indivíduos adolescentes não são considerados como um grupo prioritário a ser cuidado, ainda que esta seja uma recomendação prevista em lei, no artigo 227 da Constituição Federal (BRASIL, 1988). O fato reforça ainda mais a relevância das intervenções de Enfermagem (MATIAS et al., 2013).

Diante dessa abordagem, o estudo objetivou descrever os resultados alcançados a partir da experiência de promoção e educação em saúde com adolescentes matriculados em uma instituição pública de ensino na região Centro- Oeste do Brasil.

\section{MATERIAL E MÉTODOS}

Estudo descritivo consistiu em um relato de experiência, modalidade de estudo baseado na memorização e registro de experiências, pertencente à mesma natureza das biografias, notícias, testemunhos e outros (ELIAS, 2014). O presente relato é oriundo de oficinas com adolescentes, desenvolvidas por acadêmicos de enfermagem, estagiários em uma Unidade Básica de Saúde/Estratégia Saúde da Família, no período de maio a junho de 2016. O cenário foi uma escola pública da cidade de Catalão, estado de Goiás, região Centro-Oeste do Brasil.

No total, estiveram presentes nas oficinas, 161 adolescentes, com idades entre 13 e 17 anos, de ambos os sexos e cursando o oitavo ano do ensino médio. Em cada encontro, participaram em média, 40 alunos.

As ações desenvolveram-se em quatro encontros, realizados quinzenalmente, com duração de aproximadamente 90 minutos. Em cada oficina foi abordado um tema específico, a ordem dos temas seguiu as orientações e preferências das responsáveis pedagógicas da instituição. Sendo assim, o primeiro encontro foi intitulado "Doenças Sexualmente Transmissíveis e Métodos Contraceptivos"; já o segundo, "Transtornos Alimentares e Gripe Suína"; o terceiro, "Uso e abuso de substâncias psicoativas" e o último " Transformações corporais". As técnicas de ensino e aprendizagem utilizadas foram exposição de slides dinâmicos, documentários, vídeos e conversa/debate. 
Destaca-se que a escolha da modalidade oficina se deu em virtude de ser uma estratégia que favorece a aprendizagem compartilhada. É desenvolvida por meio de atividade grupal, com a intenção de oferecer um espaço acolhedor aos participantes, visando o empoderamento e a criatividade na busca de soluções pessoais, familiares, comunitárias e de transformação da realidade (BASTIANI \& PADILHA, 2007).

Para a execução das oficinas empregou-se a metodologia participativa, tendo em vista que esta abordagem permite o envolvimento do sujeito no processo da construção do conhecimento. Ressalta-se que esta metodologia estimula a reflexão e a participação, considerando que estes aspectos fomentam a compreensão dos temas (FONSECA et al., 2010).

Ao final de cada encontro, os acadêmicos de enfermagem realizaram perguntas simples referentes aos temas discutidos no dia para avaliar 0 conhecimento adquirido pelos adolescentes e a eficácia da metodologia aplicada.

\section{RESULTADOS E DISCUSSÃO}

No início de cada encontro, após a apresentação dos acadêmicos responsáveis pelas oficinas, os mesmos indagaram aos adolescentes quanto aos seus conhecimentos a respeito de cada assunto a ser discutido. Assim, foi possível observar que os temas mais desconhecidos eram "DST" e "Métodos Contraceptivos", haja vista que os adolescentes não sabiam, sequer, o significado dessas palavras. Tal constatação demonstra a vulnerabilidade do público frente à contaminação e transmissão das DSTs, assim como à gestação não planejada.

Participantes de outros estudos também manifestaram pouca compreensão dos conteúdos referidos, demonstrando constrangimento ao debater sobre comportamento sexual. $O$ fato dos adolescentes não conhecerem o próprio corpo justifica e agrava a situação, pois, isso os tornam incapazes de reconhecer a sintomatologia e as formas de contágio das DSTs. A vulnerabilidade apresentada relaciona-se, ainda, com a sensação de domínio próprio e autoestima elevada, que favorecem a exposição às situações de risco (BRÊTAS, 2010; LUNA et al., 2012).

Em geral, todos os participantes demonstraram interesse em aprender, sendo este revelado por meio de dúvidas e interrupções para comentários. Nesse sentido, quanto aos métodos contraceptivos e a gravidez, as expressões de surpresa e espanto sobressaíram em todos os grupos. Explicado o significado de método contraceptivo, o único citado pelos estudantes foi o preservativo masculino. Autores afirmam que a baixa procura e adesão aos métodos contraceptivos estão associadas ao conhecimento insuficiente acerca dos mecanismos existentes, a má informação, a ideia de que o contraceptivo atrapalha no prazer sexual, a baixa autoestima que faz com que o adolescente não se preocupe em se proteger, e, por fim, a sensação de invulnerabilidade (SILVA et al., 2013).

No tocante aos temas "Transtornos Alimentares e Gripe Suína", o grupo demonstrou interatividade, entendimento prévio e identificação com alguns dos aspectos explorados. Verificou-se que cinco adolescentes apresentavam transtornos alimentares, sendo três deles com obesidade e dois com anorexia, ambos os transtornos, constatados por meio do relato dos próprios acometidos. Os transtornos alimentares configuram-se como disfunções complexas que afetam os adolescentes de forma progressiva e alarmante, sugerindo a 
necessidade de constantes intervenções para conscientizar sobre os riscos comportamentais e medidas preventivas que promovam qualidade alimentar e minimizem as consequências dos hábitos de vida pouco saudáveis (PEDRINOLA, 2012; CUBRELATI et al., 2014; BREVIDELLI et al., 2015).

O excesso na ingestão de carboidratos e a inatividade física são comportamentos comuns na adolescência. Estes se estabelecem como principais fatores que colaboram para o excesso de peso nessa fase da vida, sendo que os três adolescentes que relataram ser obesos reconhecem a ingestão de carboidratos e a inatividade física como fatores de risco para a obesidade. A doença em questão é a mais prevalente entre adolescentes no Brasil, configurando uma situação preocupante que expõe os jovens ao risco para doenças cardiovasculares e musculoesqueléticas no decorrer da vida (CABRERA et al., 2014 : PINHO et al., 2014). Já a anorexia, presente em dois dos participantes das oficinas, decorre do momento de transição vivido e da necessidade de se enquadrar nos padrões de beleza. Igualmente aos outros transtornos da alimentação, a anorexia requer intervenção imediata e eficaz, pois pode acarretar consequências críticas à saúde (OLIVEIRA et al., 2013).

Ao abordar os temas Gripe-Influenza e Drogas ficou evidente que os adolescentes obtêm bastante informações, fazendo desses temas os mais conhecidos pelos participantes das oficinas. Entretanto, percebeU-se que apesar de terem conhecimento sobre os assuntos, os adolescentes ainda apresentam dificuldades para transformar as informações em ações (ADAMCHESKI et al., 2015).

As formas de transmissão e prevenção, bem como o quadro clínico da Influenza foram elementos expostos de forma satisfatória pelos participantes, deixando de mencionarem apenas, o agente etiológico e o modo de tratar a doença. Nessa perspectiva, foram informados sobre os tópicos não respondidos, e também orientados quanto a algumas ideias errôneas em relação ao assunto, como a de que o nome popular "gripe suína" indica que a patologia é transmitida pelo porco. Ademais, enfatizou-se as condutas preventivas que devem ser tomadas no ambiente escolar e familiar, levando em consideração que implementar estratégias educativas é a melhor ferramenta capaz de gerar efeitos positivos (ADAMCHESKI et al., 2015).

Ao apresentar a temática das drogas, foi comum em todas as turmas 0 conhecimento prévio em relação a essas substâncias. Os adolescentes souberam distinguir drogas lícitas e ilícitas, citaram alguns dos tipos de drogas existentes, sendo mais conhecidas, a maconha e o crack; e, demonstraram saber os principais motivos do uso e as consequências advindas deste. Cabe ressaltar que um fato observado por meio de algumas falas foi a banalização do álcool e do tabaco, pois, por serem lícitas no Brasil, são consideradas inofensivas ou "drogas leves" por alguns dos estudantes. "Se for assim, todo mundo já usou droga na vida" é um trecho cuja interpretação revela o quanto esse tipo de substância é comum ou até "normal" na perspectiva do público estudado (LAMARCA \& VETTORE, 2013).

Estudos similares mostram que além de serem consideradas comuns, as drogas lícitas são as mais utilizadas por adolescentes. Apontando este dado como preocupante, as investigações sugerem que os trabalhos preventivos desenvolvidos nas escolas devem manter o foco especialmente para o álcool. Além disso, muitos adolescentes citaram que têm pais dependentes de álcool, tabaco ou ambos, estando expostos ao início do uso, já que é conhecida a 
chance elevada de uso dessas drogas por adolescentes que convivem com pais usuários (CARLINI et al., 2010 : ELICKER et al., 2015).

Em relação às transformações corporais, é prudente mencionar o nível de conhecimento insatisfatório observado. Entre todas as mudanças que ocorrem na puberdade, apenas desenvolvimento das mamas e crescimento de pelos foram mencionados pelos participantes. Nesse contexto, esclareceu-se a respeito de cada uma das modificações decorrentes do processo de maturação sexual (MANTOVANI et al., 2014).

A falta de informação presente entre os adolescentes é atribuída à ausência de políticas e programas que implementem ações educativas voltadas aos jovens. Acrescenta-se ainda que trabalhar questões referentes à sexualidade com adolescentes requer preparo e habilidade, o que em geral não acontece com os pais, educadores e profissionais da saúde, principais responsáveis pela educação, nesse contexto (FILLIPINI et al., 2013).

\section{CONCLUSÃO}

O desenvolvimento da educação em saúde se mostrou como oportunidade de sensibilização, aprendizado e autoconhecimento para os adolescentes, que vivenciam uma fase de muitas dúvidas e descobertas. Por meio de perguntas no final de cada encontro acerca do tema discutido na oficina, constatou-se que os jovens envolvidos demonstraram insuficiência de informações de saúde, assim, as ações desenvolvidas se mostraram como uma ferramenta conveniente e eficaz para promover mudanças benéficas, diminuir suas vulnerabilidades e auxiliar no bom enfrentamento das dificuldades presentes nesse período da vida.

Neste sentido, percebeu-se que a escola é um ambiente favorável para a execução de atividades desse cunho, na medida em que, por ser um local onde os adolescentes passam grande tempo do dia, o alcance dos objetivos se torna mais fácil. Além disso, este já se caracteriza como um espaço para promover educação, assim, a articulação entre professores e profissionais da saúde potencializou a obtenção dos resultados.

A ação favoreceu o preparo dos acadêmicos envolvidos para assumir a função de educadores em saúde, ao passo que oportunizou a busca de conhecimento técnico e científico, assim como, permitiu ampliar o olhar sobre este público, que necessita ser cuidado como todos os grupos etários.

\section{REFERÊNCIAS}

ADAMCHESKI, J. K.; WIECZORKIEVICZ, A. M.; JUNKES, C. H. G. Imunização na adolescência: procura vacinal e outros determinantes. Saúde e Meio Ambiente Santa Catarina, v. 4, n. 2, p. 115-124, 2015.

BASTIANI, J. A. N.; PADILHA, M. I. C. S. Experiência dos Agentes Comunitários de Saúde em Doenças Sexualmente Transmissíveis. Revista Brasileira de Enfermagem, Brasília, v. 60, n. 2, p. 233-236, 2007.

BRASIL, CONSTITUIÇÃO DA REPÚBLICA FEDERATIVA DO BRASIL de 1988, Diário Oficial da União, Poder Executivo, Brasília, DF: Senado Federal, 1988. Disponível <http://www.planalto.gov.br/ccivil_03/Constituicao/Constituicao.htm>. Acesso em: 19 ago. 2016. 
Ministério da Saúde. Conselho Nacional de Secretários de Saúde. Atenção Primária e Promoção da Saúde. Brasília: CONASS, ed. 1, 2007.

Ministério da Saúde. Secretaria de Atenção à Saúde. Departamento de Atenção Básica. Saúde na Escola. Brasília: Ministério da Saúde, 2009.

BRÊTAS, J. R. S. Vulnerabilidade e adolescência. Revista da Sociedade Brasileira de Enfermeiros Pediatras, São Paulo, v.10, n.2, p.89-96, 2010.

BREVIDELLI, M. M.; COUTINHO, R. M. C.; COSTA, L. F. V.; COSTA, L. C. Prevalência e fatores associados ao sobrepeso e obesidade entre adolescentes de uma escola pública. Revista Brasileira de Promoção da Saúde, Fortaleza, v. 28. n. 3, p. 379-386, 2015.

CABRERA, T. F. C.; CORREIA, I. F. L.; SANTOS, D. O.; PACAGNELLI, F. L.; PRADO, M. T.A.; SILVA, T. D.; MONTEIRO, C. B. M.; FERNANI, D. C. G. L. Análise da prevalência de sobrepeso e obesidade e do nível de atividade física em crianças e adolescentes de uma cidade do sudoeste de São Paulo. Journal of Human Growth and Development, São Paulo, v. 24, n.1, p. 67-66, 2014.

CARLINI, E. L. A. CEBRID. VI Levantamento Nacional sobre o Consumo de Drogas Psicotrópicas entre Estudantes de Ensino Fundamental e Médio das Redes Pública e Privada de Ensino nas 27 Capitais Brasileiras. CEBRID - Centro Brasileiro de Informações sobre Drogas Psicotrópicas: UNIFESP Universidade Federal de São Paulo, São Paulo, 2010.

CEDARO, J. J.; VILAS BOAS, L. M. S.; MARTINS, R. M. Adolescência e Sexualidade: Um Estudo Exploratório em uma Escola de Porto Velho-RO. Psicologia: Ciência e Profissão, v. 32, n. 2, p. 320-339, 2012.

COSSA, A. P. P.; JARDIM, D. P. O enfermeiro na educação em saúde na adolescência nos últimos dez anos. Revista de Enfermagem da UNISA, São Paulo, v. 12, n. 1, p. 58-63, 2011.

COSTA, A. C. P. J.; ARAÚJO, M. F. M.; ARAÚJO, T. M.; GUBERT, F. A.; VIEIRA, N. F. C. Protagonismo de adolescentes na prevenção de doenças sexualmente transmissíveis. Acta Paulista de Enfermagem, São Paulo, v. 28, n. 5, p. 482-487, 2015.

COSTA, G. M. C.; CAVALCANTI, V. M.; BARBOSA, M. L.; CELINO, S. D. M.; FRANÇA, I. S. X.; SOUSA, F. S. Promoção de saúde nas escolas na perspectiva de professores do ensino fundamental. Revista Eletrônica de Enfermagem, Goiás, v. 15, n. 2, p. 506-515, 2013.

COUTINHO, I. R. A. B.; MARQUES, J. O.; SANTOS, J. V. M.; PINTO, D. S.; BARBOSA, W. A. S. Promoção da educação em saúde na adolescência através de oficinas temáticas. Revista de Ciências da Saúde Nova Esperança, Paraíba, v. 13, n. 2, p. 88-93, 2015.

CUBRELATI, B. S.; RIGONI, P. A. G.; VIEIRA, L. F.; BELEM, I. C. Relação ENCICLOPÉDIA BIOSFERA, Centro Científico Conhecer - Goiânia, v.13 n.24; p.1859 
entre distorção de imagem corporal e risco de desenvolvimento de transtornos alimentares em adolescentes. Conexões, Campinas, v. 12, n. 1, p. 1-15, 2014.

ELIAS, L. M. Como escrever um bom Relato de Experiência em "Implantação de Sistema de Informações de Custos no setor público". In: I Seminário Regional de informação de custos e qualidade do gasto no setor público Região Norte. FIEBA. Belém, 2014.

ELICKER, E.; PALAZZO, L. S.; AERTS, D. R. G. C.; ALVES, G. G.; CÂMARA, S. Uso de álcool, tabaco e outras drogas por adolescentes escolares de Porto Velho- RO, Brasil. Epidemiologia e Serviços de Saúde, Brasília, v. 24, n. 3, p. 399-410, 2015.

FALKENBERG, M. B.; MENDES, T. P. L.; MORAES, E. P.; SOUZA, E. M. Educação em saúde e educação na saúde: conceitos e implicações para a saúde coletiva. Ciência \& Saúde Coletiva, Rio de Janeiro, v. 19, n. 3, p. 847-852, 2014.

FILLIPINI, C. B.; PRADO, B. O; FELIPE A. O. B.; TERRA, F. S. Transformações físicas e psíquicas: um olhar do adolescente. Adolescência e Saúde, Rio de Janeiro, v. 10, n. 1, p. 22-29, 2013.

FONSECA, A. D.; GOMES, V. L. O.; TEIXEIRA, K. C. Percepção de adolescentes sobre uma ação educativa em orientação sexual realizado por acadêmicos (as) de enfermagem. Escola Anna Nery Revista de Enfermagem, Rio de Janeiro, v. 14, n. 2, p. 330-337, 2010.

LAMARCA, G.; VETTORE, M. Uso de substâncias lícitas entre os adolescentes no Brasil [Internet]. Rio de Janeiro: Portal DSS Brasil, 2013. Disponível em: http://dssbr.org/site/?p=14803\&preview=true

LUNA, I. T.; SILVA, K. L.; DIAS, F. L. A.; FREITAS, M. M. C.; VIEIRA, N. F. C.; PINHEIRO, P. N. C. Ações educativas desenvolvidas por enfermeiros brasileiros com adolescentes vulneráveis às DST/AIDS. Ciencia y Enfermería, Concepción, v. 18, n. 1, p. 43-55, 2012.

MANTOVANI, G. D.; TRES, B.; SILVA, R. M. M.; MOURA, C. B. Comparação de Dúvidas Sobre Sexualidade Entre Crianças e Adolescentes. Contexto e Educação, Rio Grande do Sul, v. 29, n. 92, p. 72-90, 2014.

MATIAS, E. O.; SOUSA, C. N. S.; NEVES, C. S.; CARNEIRO, J. L.; BRITO, L. M. S.; MELO, K. M. Estratégia educativa como tecnologia facilitadora para promoção da saúde do adolescente no âmbito escolar. Adolescência e Saúde, Rio de Janeiro, v. 10, n. 2, p. 7-14, 2013.

OLIVEIRA, J. G.; CARVALHO, B. R. A.; ROSA, H. C. S.; SANTOS, L. E. L.; MOURA, T. A.; MOREIRA, N. S. Anorexia nervosa na adolescência e suas consequências na imagem corporal: um olhar psicanalítico. Cadernos Brasileiros de Saúde Mental, Florianópolis, v.5, n.12, p. 40-59, 2013. 
ORGANIZAÇÃO MUNDIAL DA SAÚDE. Saúde do Adolescente. Geneva: WHO, 2016. Disponível em: <http://www.who.int/topics/adolescent_health/es/>. Acesso em: 18 ago. 2016.

PEDRINOLA, F. Nutrição e transtornos alimentares na adolescência. Pediatria Moderna, São Paulo, v. 8, n. 7, p. 290-295, 2012.

PINHO, L.; FLÁVIO, E. F.; SANTOS, S. H. S.; BOTELHO, A. C. C.; CALDEIRA, A. P. Excesso de peso e consumo alimentar em adolescentes de escolas públicas no norte de Minas Gerais, Brasil. Ciência \& Saúde Coletiva, Rio de Janeiro, v. 19, n. 1, p. 67-74, 2014.

SIERRA, V. M.; MESQUITA, W. A. Vulnerabilidades e fatores de risco na vida de crianças e adolescentes. São Paulo em Perspectiva, São Paulo, v. 20, n. 1, p. 148- 155, 2006.

SILVA, J. M. Q.; MARQUES, P. F.; PAIVA, M. S. Saúde sexual e reprodutiva e enfermagem: um pouco de história na Bahia. Revista Brasileira de Enfermagem, Brasília, v. 66, n. 4, p. 501-507, 2013. 